\title{
On the Forgetten Topological Index and Co Index
}

\author{
Gülistan Kaya Gök ${ }^{1 *}$ \\ ${ }^{1}$ Hakkari University, Department of Mathematics Education, Hakkari, Turkey (ORCID: 0000-0001-9059-1606)
}

(First received 3 January 2019 and in final form 5 March 2019)

(DOI: 10.31590/ejosat.507773)

ATIF/REFERENCE: Gök, G. K. (2019). On the Forgetten Topological Index and Co Index. European Journal of Science and Technology, (15), 308-314.

\begin{abstract}
The forgotten topological index of $\mathrm{G}$ is specified the degrees $d_{i}$ and $d_{j}$. In this paper, we find some bounds for the eigenvalues of forgotten topological matrix and we establish some inequalities about forgotten Estrada topological index.
\end{abstract}

Keywords: Forgotten topological index, Estrada index.

\section{Unutulmuş Topolojiksel İndeks ve Eş İndeks}

$\ddot{\mathbf{O} z}$

G’ nin unutulmuş topolojiksel indeksi $d_{i}$ ve $d_{j}$ dereceleriyle tanımlanır. Bu makalede, unutulmuş topolojiksel indeksin özdeğerleri için bazı sınırlar bulunmuştur ve unutulmuş Estrada topolojiksel indeks ile ilgili bazı eşitsizlikler belirlenmiştir.

Anahtar Kelimeler: Unutulmuş topolojiksel indeks, Estrada indeks.

* Corresponding Author: Hakkari University, Department of Mathematics Education, Hakkari, Turkey, ORCID: 0000-0001-9059-1606, gulistankayagok@hakkari.edu.tr 


\section{Introduction}

Let $\mathrm{G}$ be a simple, connected graph on the vertex set $\mathrm{V}(\mathrm{G})$ and the edge set $\mathrm{E}(\mathrm{G})$. For $v_{i} \in V(G)$, the degree of the vertex $v_{i}$ denoted by $d_{i}$.

The $[F](G)$ forgotten topological matrix of graphs is defined as

$$
[F]_{i j}=\left\{\begin{array}{cc}
d_{i}^{2}+d_{j}^{2} & \text { if } i \text { adjacent to } j \\
0 & \text { otherwise. }
\end{array}\right.
$$

The eigenvalues of $[F](G)$ is denoted by $f_{i}, i=1,2, \ldots, n$. Considering these eigenvalues, we apply some known lemmas and we obtain some bounds including the first eigenvalue. In addition, we set some conclusions using the complement of the first eigenvalue.

The Forgotten Topological Index ( $F$ index) of $\mathrm{G}$ is described in [4], [5] as

$$
\sum_{v_{i} v_{j} \in E(G)} d_{i}^{2}+d_{j}^{2}
$$

The Estrada index of the graph $\mathrm{G}$ in [3], [9] as

$$
E E(G)=\sum_{i=1}^{n} e^{\lambda_{i}}
$$

where $\lambda$ is the eigenvalue of the adjacency matrix of $\mathrm{G}$.

The plan of this paper is as follows: In section 2, we give some known lemmas. In section 3, we have some inequalities in terms of the degrees, the edges and the vertices. Also, we define forgotten Estrada topological index and we find different relations concepting these indices and its eigenvalues.

(See [1], [6], [8] for more details.)

\section{Preliminaries}

In this section, we will give some lemmas and theorems to be utilized in main results.

Lemma 2.1. [7]

Let $M=\left(m_{i j}\right)$ be an $n x n$ irreducible nonnegative matrix and $\lambda_{1}(M)$ be the greatest eigenvalue with $R_{i}(M)=\sum_{j=1}^{m} m_{i j}$. Then,

$$
\left(\operatorname{minR}_{i}(M): 1 \leq i \leq n\right) \leq \lambda_{1}(M) \leq\left(\max R_{i}(M): 1 \leq i \leq n\right)
$$

Lemma 2.2. [2]

If $G$ is a simple, connected graph and $\lambda_{1}(G)$ is the spectral radius, then

$$
\lambda_{1}(G) \leq \max \left(\sqrt{m_{i} m_{j}}: 1 \leq i, j \leq n, v_{i}, v_{j} \in E\right) .
$$

\section{Main Results}


Firstly, we set a relation for the largest eigenvalue of $F$ matrix including the degrees, the edges and the vertices. After, we will set some inequalities concerned with F Estrada index.

\section{Definition 3.1.}

The Estrada $F$ index of the graph $\mathrm{G}$ is described as

$$
E^{F}(G)=\sum_{i=1}^{n} e^{f_{i}}
$$

where $f_{1} \geq f_{2} \geq \cdots \geq f_{n}$ are the eigenvalues of $[F](G)$.

\section{Definition 3.2.}

The Estrada $F$ coindex of the graph $\mathrm{G}$ is specified as

$$
E^{F}(G)=\sum_{i=1}^{n} e^{\bar{f}_{l}}
$$

where $\overline{f_{1}} \geq \overline{f_{2}} \geq \cdots \geq \overline{f_{n}}$ are the eigenvalues of the complement of $[F](G)$.

\section{Theorem 3.1.}

If $G$ is a simple, connected graph then

$$
f_{1}(G) \leq \sqrt{\left(n d_{i}^{2}+4 m^{2}\right)\left(n d_{j}^{2}+4 m^{2}\right)} .
$$

Proof. Let $D(G)^{-1} F(G) D(G)=F^{+}(G)$ and $X=\left(x_{1}, x_{2}, \ldots, x_{n}\right)^{T}$ be an eigenvector of $F^{+}(G)$. Also, $x_{i}=1$ and $0<x_{i} \leq 1$ for every $k$. Let $x_{j}=\max _{k}\left(x_{k}: v_{i} v_{k} \in E\right)$ where $i$ is adjacent to $k$. Let $F^{+}(G) X=f_{1}(G) X$. If we get $i$-th equation from above equation, then

$$
f_{1}(G) x_{i}=\sum_{k}\left(d_{i}^{2}+d_{k}^{2}\right) x_{k} \leq\left(n d_{i}^{2}+(2 m)^{2}\right) x_{k}
$$

By Lemma 2.1, we have

$$
f_{1}(G) x_{i} \leq\left(n d_{i}^{2}+4 m^{2}\right) x_{k}
$$

The $j$-th equation of the same equation,

$$
f_{1}(G) x_{j} \leq\left(n d_{j}^{2}+4 m^{2}\right) x_{k}
$$

From Lemma 2.2,

$$
f_{1}(G) \leq \sqrt{\left(n d_{i}^{2}+4 m^{2}\right)\left(n d_{j}^{2}+4 m^{2}\right)} .
$$

\section{Theorem 3.2.}

Let $\mathrm{G}$ be a graph on $\mathrm{n}$ vertices and $\mathrm{m}$ edges. Then, 


$$
\left(\bar{f}_{1}(G)\right) \leq \sqrt{\mathcal{L}-\left(n d_{i}^{2}+4 m^{2}\right)\left(n d_{j}^{2}+4 m^{2}\right)}
$$

where $\quad \mathcal{L}=\left(d_{i}^{2}+d_{j}^{2}\right)\left[(n-1)^{2}+n^{3}(n-1)^{2}-4 m n^{2}(n-1)\right]+2 n^{2} d_{i} d_{j}\left[d_{i} d_{j}+2(n-1)^{2}\right]-\left(2\left(d_{i}+d_{j}\right)(n-1)\right)[((n-$ 1) $\left.n)^{2}+d_{i} d_{j}+n^{2} m(n-1)^{2}-4 m n(n-1)+4 m^{2}\right]+(n-1)^{4} n^{2}\left[2 m+n^{2}\right]+(n-1)^{3} m n\left[-8-\frac{n^{2}}{2}\right]+(n-1)^{2} m^{2}[8 m+$ $\left.2 n^{2}-\frac{n^{2}}{2}\right]-2 m^{3} n(n-1)+2 m^{4}$.

Proof. Cauchy-Schwarz inequality gives that

$$
\begin{gathered}
\left(f_{1}(G)+\bar{f}_{1}(G)\right)^{2} \leq\left(f_{1}(G)\right)^{2}+\left(\bar{f}_{1}(G)\right)^{2} \\
\leq\left(n d_{i}^{2}+4 m^{2}\right)\left(n d_{j}^{2}+4 m^{2}\right)+\left(\left(n-1-d_{i}\right)^{2}+4 \bar{m}^{2}\right)\left(\left(n-1-d_{j}\right)^{2}+4 \bar{m}^{2}\right) .
\end{gathered}
$$

It is implies that

$$
\begin{gathered}
\left(f_{1}(G)\right)^{2}+\left(\bar{f}_{1}(G)\right)^{2} \leq\left(n^{2} d_{i}^{2} d_{j}^{2}+4 m^{2} n\left(d_{i}^{2}+d_{j}^{2}\right)+16 m^{4}\right)+\left(n^{2}\left(n-1-d_{i}\right)^{2}\left(n-1-d_{j}\right)^{2}\right. \\
\quad+4 \bar{m}^{2} n\left[\left(n-1-d_{i}\right)^{2}+\left(n-1-d_{j}\right)^{2}\right]+16 \bar{m}^{4} \\
=\left(n^{2}\left(\left(n-1-d_{i}\right)^{2}\left(n-1-d_{j}\right)^{2}+d_{i}^{2} d_{j}^{2}\right)+16\left[m^{4}+\bar{m}^{4}\right]\right. \\
\left.+4 n\left(d_{i}^{2}+d_{j}^{2}\right)\left(m^{2}+\bar{m}^{2}\right)+8 \bar{m}^{2} n\left[(n-1)-d_{i}-d_{j}\right)\right] .
\end{gathered}
$$

Since $m+\bar{m}=\frac{n^{2}-n}{2}$ then,

$$
\begin{gathered}
m^{4}+\bar{m}^{4}=\left(m^{2}+\bar{m}^{2}\right)^{2}-2 m^{2} \bar{m}^{2} \\
=\left((m+\bar{m})^{2}-2 m \bar{m}\right)^{2}-2(m \bar{m})^{2} \\
=\left(\left(\frac{n^{2}-n}{2}\right)^{2}-2 m\left(\frac{n^{2}-n}{2}-m\right)\right)^{2}-2\left(m\left(\frac{n^{2}-n}{2}-m\right)\right)^{2} .
\end{gathered}
$$

So, we get

$$
\begin{gathered}
\left(f_{1}(G)\right)^{2}+\left(\bar{f}_{1}(G)\right)^{2} \leq\left(d_{i}^{2}+d_{j}^{2}\right)\left[(n-1)^{2}+n^{3}(n-1)^{2}-4 m n^{2}(n-1)\right] \\
+2 n^{2} d_{i} d_{j}\left[d_{i} d_{j}+2(n-1)^{2}\right]-2\left(d_{i}+d_{j}\right)(n-1) \\
+\left[((n-1) n)^{2}+d_{i} d_{j}+n^{2} m(n-1)^{2}-4 m n(n-1)+4 m^{2}\right] \\
+(n-1)^{4} n^{2}\left[2 m+n^{2}\right]+(n-1)^{3} m n\left[-8-\frac{n^{2}}{2}\right] \\
+(n-1)^{2} m^{2}\left[8 m+2 n^{2}-\frac{n^{2}}{2}\right]-2 m^{3} n(n-1)+2 m^{4}
\end{gathered}
$$

It is conclude that,

$$
\left(\bar{f}_{1}(G)\right)^{2} \leq \mathcal{L}-\left(f_{1}(G)\right)^{2} .
$$


From Theorem 3.1,

$$
\left(\bar{f}_{1}(G)\right) \leq \sqrt{\mathcal{L}-\left(n d_{i}^{2}+4 m^{2}\right)\left(n d_{j}^{2}+4 m^{2}\right)} .
$$

\section{Theorem 3.3.}

Let $G$ be graph on $n$ vertices, $m$ edges and $E_{F}$ be Estrada $F$ index. Then,

$$
E_{F} \leq n e^{\sqrt{\left(n d_{i}^{2}+4 m^{2}\right)\left(n d_{j}^{2}+4 m^{2}\right)}}
$$

Proof. By Theorem 3.1, we have that

$$
E_{F}=\sum_{j=1}^{n} e^{f_{j}} \leq n e^{f_{1}}=n e^{\sqrt{\left(n d_{i}^{2}+4 m^{2}\right)\left(n d_{j}^{2}+4 m^{2}\right)}} .
$$

\section{Theorem 3.4.}

Let $G$ be graph on $n$ vertices, $m$ edges and $E_{F}$ be Estrada $F$ index. Then,

$$
E_{F} \leq \sqrt{n^{2}-(2 n-1) e^{2 \sqrt{\left(n d_{i}^{2}+4 m^{2}\right)\left(n d_{j}^{2}+4 m^{2}\right)}}}-e^{\sqrt{\left(n d_{i}^{2}+4 m^{2}\right)\left(n d_{j}^{2}+4 m^{2}\right)}} .
$$

Proof. We know that

$$
\begin{gathered}
\left(E_{F}-e^{f_{n}}\right)^{2}=E_{F}{ }^{2}-2 E_{F} e^{f_{n}}+\left(e^{f_{n}}\right)^{2} \\
=\left(\sum_{j=1}^{n} e^{f_{j}}\right)^{2}-2\left(\sum_{j=1}^{n} e^{f_{j}}\right) e^{f_{n}}+e^{2 f_{n}}
\end{gathered}
$$

By the Arithmetic-Geometric Mean inequality, we result that

$$
\left(\sum_{j=1}^{n} e^{f_{j}}\right)^{2} \leq\left(n\left(\prod_{j=1}^{n} e^{f_{j}}\right)^{\frac{1}{n}}\right)^{2}=n^{2}\left(e^{\sum_{j=1}^{n} e^{f_{j}}}\right)^{\frac{2}{n}}
$$

Since $f_{1} \geq f_{2} \geq \cdots \geq f_{n}$ then,

$$
\begin{gathered}
\left(E_{F}-e^{f_{n}}\right)^{2} \leq n^{2}-2 n e^{f_{n}} e^{f_{n}}+e^{2 f_{n}} \\
=n^{2}-(2 n-1) e^{2 f_{n}} \\
=n^{2}-(2 n-1) e^{2 \sqrt{\left(n d_{i}^{2}+4 m^{2}\right)\left(n d_{j}^{2}+4 m^{2}\right)}} .
\end{gathered}
$$


It gives that

$$
E_{F} \leq \sqrt{n^{2}-(2 n-1) e^{2 \sqrt{\left(n d_{i}^{2}+4 m^{2}\right)\left(n d_{j}^{2}+4 m^{2}\right)}}}+e^{\sqrt{\left(n d_{i}^{2}+4 m^{2}\right)\left(n d_{j}^{2}+4 m^{2}\right)}} .
$$

\section{Theorem 3.5.}

Let $G$ be graph on $n$ vertices, $m$ edges and $E_{F}$ be Estrada $F$ index. Then,

$$
E_{F}+E_{\bar{F}} \leq \sum_{k=0}^{\infty} \frac{n \sqrt{\mathcal{L}}}{k !}
$$

where $E_{\bar{F}}$ is Estrada $F$ coindex of G.

Proof. By the sum of the Estrada $F$ index and Estrada $F$ coindex, we find that

$$
\begin{aligned}
E_{F}+E_{\bar{F}} & =\left(\sum_{j=1}^{n} e^{f_{j}}\right)+\left(\sum_{j=1}^{n} e^{\bar{f}_{J}}\right) \\
& =\sum_{k=0}^{\infty} \frac{1}{k !}\left(\sum_{j=1}^{n}\left(f_{j}+\bar{f}_{J}\right)\right) \\
& \leq \sum_{k=0}^{\infty} \frac{n}{k !}\left(f_{j}+\bar{f}_{J}\right) .
\end{aligned}
$$

Using Theorem 3.2, we obtain

$$
E_{F}+E_{\bar{F}} \leq \sum_{k=0}^{\infty} \frac{n \sqrt{\mathcal{L}}}{k !}
$$

\section{Conclusions}

In this study, we expand forgotten topological index, we define F Estrada index and we find some bounds deal with this index. In the sequel, we describe F Estrada coindex and we obtain an inequalities for this index.

\section{Acknowledge}

The author would like thank for the valuable suggestions of referees. 


\section{References}

[1] Büyükköse, S., Kaya Gök, 2018. G. Graf Teoriye Giriş, Nobel Akademik Yayıncılık Eğitim Danışmanlık Tic. Ltd. Sti, Ankara.

[2] Das, K. C., Kumar, P. 2004. Some new bounds on the spectral radius of graphs. Discrete Mathematics (281), $149-161$.

[3] Estrada, E. 2000. Characterization of 3D molecular structure. Chem. Phys. Lett. (319), 713-718.

[4] Furtula, B., Gutman I. 2015. A forgotten topological index. J. Math. Chem. (53), 1184-1190.

[5] Gutman I. 2014. On the origin of two degree-based topological indices. Bull. Acad. Serb. Sci. Arts (Cl. Sci. Math. Natur.) (146), 39-52.

[6] Kaya Gök, G. 2018. Some bounds on the distance-sum-connectivity matrix. Journal of Inequalities and Applications. 171.

[7] Horn, R.A., Johnson, C.R. 1985. Matrix Analysis. Cambridge University Press, New York.

[8] Sorgun, S., Büyükköse, Ş. 2012. The new upper bounds on the spectral radius of weighted graphs. Applied Mathematics and Computation. (218), 5231-5238.

[9] Du, Z., Zhongzhu, L. 2011. On the Estrada and Laplacian Estrada indices of graphs. Linear Algebra and its Applications. (435), 2065-2076. 